\title{
Hypernatremia in the geriatric population
}

\author{
Maulin K Shah' \\ Biruh Workeneh ${ }^{1,2}$ \\ George E Taffet ${ }^{1,3}$ \\ 'Department of Internal Medicine, \\ 2Department of Nephrology, \\ ${ }^{3}$ Huffington Center on Aging, \\ Baylor College of Medicine, \\ Houston, TX, USA
}

This article was published in the following Dove Press journal:

Clinical Interventions in Aging

19 November 2014

Number of times this article has been viewed

\begin{abstract}
Hypernatremia in the geriatric population is a common disorder associated with significant morbidity and mortality. Older people are predisposed to developing hypernatremia because of age-related physiologic changes such as decreased thirst drive, impaired urinary concentrating ability, and reduced total body water. Medications may exacerbate this predisposition. Hypernatremia and dehydration occurring in nursing homes are considered indicators of neglect that warrant reporting, but there are other nonavoidable causes of hypernatremia, and consideration at time of presentation is essential to prevent delay in diagnosis and management. We describe a case illustrating the importance of the consideration of alternate explanations for hypernatremia in a nursing home resident, followed by a review of hypernatremia in the elderly population, to underscore that neglect is the etiology of exclusion after alternatives have been considered.
\end{abstract}

Keywords: geriatric, hypernatremia, sodium

\section{Case introduction}

$\mathrm{Mr} \mathrm{H}$ is a 70-year-old black male with a history significant for dementia associated with chronic hydrocephalus. He also had urinary outflow obstruction, producing chronic bilateral hydronephrosis requiring a chronic indwelling urinary catheter. His family noticed decreased interactions and decreased oral intake developing over the course of 2 weeks. In the facility, he was found to have a urinary tract infection and was treated with sulfamethoxazole/trimethoprim without improvement in his symptoms. Subsequent laboratory investigation revealed a serum sodium ( $\mathrm{SNa}$ ) level of $177 \mathrm{mmol} / \mathrm{L}$, and the patient was administered intravenous fluids and admitted to our hospital. His other medications on admission were bisacodyl and docusate. On admission, he was a cachectic, chronically ill-appearing man who was in no acute distress. He was tachycardic at 110 beats per minute; his vital signs were otherwise normal. He had dry mucous membranes. He had a $5 \times 4 \times 4 \mathrm{~cm}$ sacral decubitus ulcer with necrotic edges. His urinary drainage bag had clear dilute yellow urine with dark sediment. He was not interactive on mental status examination. The rest of the physical examination was normal.

Laboratory studies (roughly 8 hours after nursing home labs were drawn) revealed a white blood cell count of $18,500, \mathrm{SNa}$ of $167 \mathrm{mmol} / \mathrm{L}, \mathrm{K}$ of $3.5 \mathrm{mmol} / \mathrm{L}$, and $\mathrm{Cl}$ of $139 \mathrm{mmol} / \mathrm{L}$; blood urea nitrogen level was $56 \mathrm{mg} / \mathrm{dL}$, and creatinine level was $2.3 \mathrm{mg} / \mathrm{dL}$. Urinalysis revealed a specific gravity of 1.016 and confirmed the presence of a urinary tract infection.

Initially, the patient was diagnosed with hypovolemic hypernatremia secondary to urinary tract infection and sepsis in the setting of impaired access to free water in the facility, and there was a strong suspicion of neglect by the facility. He was treated with broadspectrum intravenous antibiotics. Because he had past admissions for hypernatremia 
Table I Intensive care unit sodium and fluid balance flow sheet

\begin{tabular}{|c|c|c|c|c|c|}
\hline Day & Sodium, mmol/L & Intake, mL & Output, mL & Net I/O, mL & Fluids \\
\hline 1 & $|45-| 46$ & 4,615 & 1,915 & $+2,700$ & $1 / 2 N S$ \\
\hline 2 & $146-150$ & 2,525 & 2,025 & +500 & D5W \\
\hline 3 & 148 & 4,725 & 3,025 & $+1,700$ & D5W \\
\hline 4 & 147 & 3,195 & 3,050 & +145 & D5W \\
\hline 5 & 146 & 2,185 & 2,770 & -585 & D5W \\
\hline 6 (day of water-deprivation test) & $\mid 48-153$ & 1,995 & 1,990 & +5 & D5W \\
\hline 7 & $|50-| 5 \mid$ & 3,935 & 3,305 & +630 & D5W \\
\hline
\end{tabular}

Abbreviations: $1 / 2$ NS, $0.45 \%$ normal saline; D5W, $5 \%$ dextrose in water; $1 / O$, intake/output.

coming from multiple different facilities, further workup was pursued. Unfortunately, the initiation of the workup was delayed, only beginning after hydration had corrected his $\mathrm{SNa}$ to $150 \mathrm{mmol} / \mathrm{L}$. At that point, he was requiring 3-4 L intravenous fluids to maintain his SNa between $145-148 \mathrm{mmol} / \mathrm{L}$, with a urine output of 3-4 L daily (Table 1). His serum osmolality was $335 \mathrm{mOsm} / \mathrm{kg}$, urine osmolality was $241 \mathrm{mOsm} / \mathrm{kg}$, urine specific gravity was $1.012-1.016$, and urine sodium was $46 \mathrm{mmol} / \mathrm{L}$, consistent with a diagnosis of diabetes insipidus. The inappropriately normal urine specific gravity and urineto-plasma osmolality ratio of 0.7 indicate water excretion despite a relative water deficit in the setting of hypernatremia. A 12 hours water deprivation test was performed (Table 2) that also documented no response to administered desmopressin, confirming a diagnosis of nephrogenic diabetes insipidus. Ultimately, his nephrogenic diabetes insipidus was managed with free water via percutaneous endoscopic gastrostomy tube; treatment with hydrochlorothiazide was not attempted because of borderline hypotension.

This case illustrates the importance of considering diabetes insipidus among other conditions when a nursing home resident presents with hypernatremia, especially recurrent hypernatremia, so that evaluation and therapy are not delayed.

\section{Introduction}

Hypernatremia is termed as a deficit of total body water relative to total body salt and is defined by a SNa concentration greater than $145 \mathrm{mmol} / \mathrm{L} .^{1,2}$ Hypernatremia is a common disorder associated with significant morbidity and mortality for older people. In nursing home residents, hypernatremia and dehydration are considered preventable and are markers of substandard care. The residents often are dependent on help to maintain adequate hydration. As hypernatremia and dehydration developing in the nursing home are considered indicators of neglect, some jurisdictions require investigation and reporting. ${ }^{3}$ This case illustrates the importance of consideration of alternate explanations for hypernatremia in a nursing home resident. The following is a review of hypernatremia focusing on the older population. Appropriate diagnosis and management in these patients can improve outcomes and prevent erroneous reporting.

\section{Hypernatremia is common and associated with high risk for morbidity and mortality in the elderly}

The prevalence of hypernatremia in older patients living in the community may be as high as $3.7 \%$, depending on the diagnostic criteria used (the range for hypernatremia threshold varied between studies from $140-150 \mathrm{mmol} / \mathrm{L}$ ). Hypernatremia is present in approximately $2 \%$ of older patients admitted to hospital, but in almost $5 \%$ of those older than 75 years in one large survey. ${ }^{4}$ Hypernatremia was present in roughly $30 \%$ of elderly patients admitted to a geriatric hospital with a febrile illness. ${ }^{5}$ Hypernatremia is a frequent complication of hospitalization. In a study involving 8,441 consecutive patients (primarily elderly) admitted to the intensive care unit, roughly $3.5 \%$ had hypernatremia on admission, and another $15 \%$ developed hypernatremia during hospitalization. ${ }^{6}$

Table 212 hour water deprivation test

\begin{tabular}{lllll}
\hline Time & $\begin{array}{l}\text { Urine osmolality } \\
\text { (mOsm/kg) }\end{array}$ & $\begin{array}{l}\text { Serum sodium } \\
(\mathbf{m m o l} / \mathbf{L})\end{array}$ & $\begin{array}{l}\text { Serum osmolality } \\
\text { (mOsm/kg) }\end{array}$ & $\begin{array}{l}\text { Urine sodium } \\
\text { (mmol/L) }\end{array}$ \\
\hline Start & 210 & 148 & 314 & 58 \\
Hour 2 & 209 & 149 & 313 & 61 \\
Hour 4 & 221 & 148 & 315 & 65 \\
Hour 6 & 220 & 151 & 321 & 67 \\
Hour 8: desmopressin given & 235 & 151 & 318 & 74 \\
Hour 10 & 233 & 153 & 321 & 66 \\
\hline
\end{tabular}


Hypernatremia is associated with high morbidity and mortality rates. In the 8,441 primarily elderly intensive care unit patients described earlier, mortality in those admitted with hypernatremia was approximately $33 \% .{ }^{6}$ In comparison, those without hypernatremia had a mortality of $15 \% .^{6}$ If the patients had normal $\mathrm{SNa}$ on admission and developed mild or moderate to severe hypernatremia while in the intensive care unit, mortality increased to $30 \%$ and $45 \%$, respectively. ${ }^{6}$ Other studies confirm these findings: mortality increases with serum sodium, and even mild hypernatremia is associated with significant mortality in hospitalized elders, even after accounting for confounders. ${ }^{6-10}$ At the time of this review, all available literature includes data in hospitalized patients; however, data about hypernatremia in the ambulatory setting are lacking. Mortality from hypernatremia does not increase with age but does increase with recurrent episodes. ${ }^{11}$ The mortality from hypernatremia may be increased by delays in diagnosis or inappropriate management. ${ }^{12}$ In fact, a previous review on hypernatremia suggests the most serious complications occur from treatment of the hypernatremia, rather than the elevated $\mathrm{SNa}$ itself. ${ }^{1}$

\section{Aging increases the risk for developing hypernatremia}

Older patients are predisposed to hypernatremia. Sodium and water balance is regulated by thirst, arginine vasopressin (AVP), and the central renin-angiotensin-aldosterone system. ${ }^{13}$ In transient hypernatremic states, thirst is such a strong stimulant for water intake that sustained hypernatremia almost never occurs unless the thirst mechanism is impaired or access to water is restricted. ${ }^{1}$ In the nursing home, both impaired thirst and limited access to water may be common challenges for the residents and may predispose them to hypernatremia. Those unable to care for themselves are more likely to have limited access to water and rely on the facility's staff to get that access. This provides the rationale for the link between dehydration/hypernatremia and substandard care. However, there are multiple studies showing that even healthy elders have an impaired thirst mechanism. Phillips et al showed that after 24 hours of water deprivation, elderly subjects were not as thirsty as younger subjects and did not drink enough water to dilute plasma to predeprivation sodium levels. ${ }^{14}$ This also held true in response to hypertonicity, produced by infusing hypertonic saline, without extracellular fluid depletion. ${ }^{15}$ In both studies, these cognitively intact elderly subjects had a thirst response that was inadequate for the degree of hypernatremia they were experiencing. ${ }^{15}$ The risk is even greater in patients with severe dementia, as many are unable to indicate their need for water. The effects of interfering with the central renin-angiotensin system or, less commonly, with AVP pathways may exaggerate the aging effects on thirst. ${ }^{16}$

Multiple studies have demonstrated a renal concentrating impairment with age. ${ }^{14,15,17-20}$ The longest nephrons, those most critical for concentrating urine, seem the most depleted with aging. ${ }^{2}$ This can lead to a decrease in sodium excretion in response to a sodium load. ${ }^{21}$ Healthy older persons can achieve a maximum urinary concentration of $700-800 \mathrm{mOsm} / \mathrm{kg}$, whereas a healthy young adult can achieve 1,200 mOsm $/ \mathrm{kg} .{ }^{2}$ After 24 hours water deprivation, elderly subjects had higher plasma osmolality and serum sodium concentration, in large part because of this inability to conserve water by concentrating the urine. These patients had higher plasma AVP levels, suggesting normal central response to osmolality and hypernatremia but an impairment in tubular response to AVP. ${ }^{14}$ This impaired responsiveness partially accounts for nocturia in older persons. ${ }^{22}$ In addition, the effects of diuretics, especially furosemide, which is commonly used in elder patients, decrease urine concentrating ability.

Lastly, this population is prone to hypernatremia because of a physiologic decrease in total body water. In young adults, water accounts approximately $60 \%-65 \%$ of body weight, whereas in older persons, it is closer to $50 \%$, and it may be even lower in women. ${ }^{22}$ Having lower total body water predisposes patients to dehydration with smaller losses of fluid, and therefore can increase the tendency to become hypernatremic.

\section{How to approach hypernatremia in a geriatric patient}

The presentation of hypernatremia in older persons is often nonspecific. The symptoms include lethargy, weakness, and in more severe cases, changes in consciousness and seizures. ${ }^{2}$ Few symptoms may be present until sodium levels exceed $160 \mathrm{mmol} / \mathrm{L} .{ }^{1}$ As noted earlier, many older patients may be hypodipsic, whereas others may have more intense thirst that diminishes as hypernatremia worsens.${ }^{1,8} \mathrm{In}$ patients with chronic hypernatremia, symptoms will be milder because of adaptation at the cellular level, especially in the central nervous system., ${ }^{1,2}$ Signs and symptoms may be even more vague in patients developing hypernatremia in the hospital. ${ }^{1}$ A recent change in consciousness is often a marker for sodium dysnatremia in the correct clinical context; however, this is a poor prognostic indicator. ${ }^{12}$

Initial workup in a patient with hypernatremia is essential because a delay in diagnosis or management can lead to higher mortality. In addition to a review of medications, 
laboratory workup should include serum electrolytes, including calcium, serum blood urea nitrogen and creatinine levels, serum and urine osmolality, and urine sodium. ${ }^{2}$ A head computed tomography scan will help, as hypernatremia induces shrinkage of brain cells, which may cause vascular rupture and lead to cerebral bleeding or subarachnoid hemorrhage. ${ }^{1,2}$

There are several approaches to hypernatremia, but it is essential to determine volume status as a prudent first step. Dry oral mucosa, abnormal supraclavicular or thigh skin turgor, absent axillary moisture, orthostasis or hypotension, and low urine output are clinical signs of hypernatremic dehydration ${ }^{12}$ but are much less useful for the elderly. Laboratory signs that suggest dehydration as a cause of hypernatremia include an elevated blood urea nitrogen level and/or creatinine level, with an increased blood urea nitrogen to creatinine ratio, metabolic alkalosis, hemoconcentration resulting in increased hematocrit, and low urine sodium. ${ }^{2}$ It is also important to exclude states such as hyperglycemia, in which excess circulatory osmoles can cause diuresis, resulting in water loss and development of hypernatremia. ${ }^{2}$ Cases of hypernatremic dehydration resulting from disorders such as diabetes insipidus are typically unmasked whenever a patient becomes incapacitated or does not readily have access to water. ${ }^{1}$ Rarely, the thirst mechanism can be interrupted as a result of medication ${ }^{23}$ or trauma, ${ }^{2}$ resulting in dysregulation and development of hypernatremia.

Adrogué et $\mathrm{al}^{1}$ and Al-Absi et $\mathrm{al}^{24}$ have described excellent comprehensive management strategies of hypernatremia, and we discuss some of the elements in the consideration of older patients. In hemodynamically unstable patients, administration of isotonic normal saline is essential, irrespective of SNa. ${ }^{1}$ In dehydrated patients, the most appropriate method for rehydration is hypotonic saline. ${ }^{1}$ Using hypotonic saline instead of isotonic normal saline is crucial, as this will aid in the correction of both volume status and sodium in a shorter time, given the constraints described here. ${ }^{1}$ In euvolemic patients, any hypotonic fluid should suffice. ${ }^{1}$ Last, hypervolemic hypernatremia is the least common type at time of hospital presentation and usually stems from excessive administration of sodium (eg, hypertonic sodium bicarbonate, hypertonic saline, etc) during the hospital course. ${ }^{24}$ In patients with hypervolemic hypernatremia, hypotonic fluid administration coupled with loop diuretics will aid in correcting volume status without worsening hypernatremia. ${ }^{24}$

Although management with the first step will invariably help acutely correct volume status and show some improvement in sodium levels, the second step requires some further considerations for ongoing treatment, including whether the hypernatremia is acute (occurred within a few hours) or chronic. If hypernatremia is unequivocally known to be acute, usually identified when symptoms develop rapidly, sodium correction can be rapid (approximately $1 \mathrm{mmol} / \mathrm{L} /$ hour), as the brain has not had enough time to adapt. ${ }^{1}$ Cell shrinkage occurs when high plasma osmolality draws water out of brain cells. ${ }^{1}$ To counteract this shrinkage, the brain cells accumulate additional solutes (initially inorganic ions, followed by organic osmolytes). ${ }^{1}$ This cellular adaptation takes $24-48$ hours. ${ }^{1}$ As a consequence, when a patient has chronic hypernatremia, to prevent cerebral edema from occurring with correction, the maximal recommended correction rate is $0.5 \mathrm{mmol} / \mathrm{L} /$ hour. ${ }^{1}$ Correcting hypernatremia too slowly also has negative consequences. Correction at $0.5 \mathrm{mmol} / \mathrm{L} /$ hour in the first 24 hours $^{25}$ and the complete correction of hypernatremia within 4 days ${ }^{11}$ improves level of cognition and mortality. Correction extended beyond 4 days may lead to permanent loss of cognitive function and higher mortality. ${ }^{11}$ Therefore, we recommend correcting hypernatremia slowly at $0.5 \mathrm{mmol} / \mathrm{L} /$ hour for the first 12-24 hours (rapid correction at $1 \mathrm{mmol} / \mathrm{L} /$ hour initially if severe symptoms are present), followed by correction to the normal range within the next 48-72 hours. If the history is unclear (the duration of hypernatremia is uncertain), a slower correction rate is likely to be safer. Attention to ongoing fluid losses and concurrent electrolyte abnormalities will improve outcomes. ${ }^{1}$ For example, hypokalemia can cause a concentrating defect in the kidney and potentially worsen dehydration. ${ }^{26,27}$

The final step for management is establishing the underlying reason for development of hypernatremia. Otherwise, the patient remains at risk for recurrence. It is crucial to consider concomitant disorders, as exemplified in the patient described in the case presented earlier.

\section{What are the causes of hypernatremia in the elderly?}

As noted earlier, the elderly have a physiologic decrease in the thirst mechanism; however, there can be a pathological decrease in free water intake as well. Primary hypodipsia results from an injury to the hypothalamus from vascular occlusion or hemorrhage, traumatic brain injury, brain tumor, or infiltrative disease. ${ }^{2}$ A computed tomography and/or magnetic resonance image of the brain can help aid in diagnosing these conditions. Another similar, but rare, entity is called "reset osmostat", in which the threshold for thirst and AVP release have been reset to a higher level. ${ }^{2}$ These patients will have a high urine osmolality. ${ }^{2}$ 
A major cause of hypernatremia in the elderly is from hypotonic fluid losses. Loop or thiazide diuretics are common medications that create a urinary concentrating defect that generates diuresis. ${ }^{2}$ Osmotic diuresis secondary to nonabsorbable solutes such as glucose (as in hyperglycemia) can create a relative concentrating defect. ${ }^{2}$ Lastly, the recovery phase from acute tubular necrosis or postobstructive nephropathy is characterized by a concentrating defect resulting from tubular injury. ${ }^{2}$ This renal concentrating defect can be identified by a urinary osmolality lower than $700 \mathrm{mOsm} / \mathrm{kg}$ and an elevated urine sodium level greater than $20 \mathrm{mmol} / \mathrm{dL} .^{2}$

Diabetes insipidus can be characterized by a severe renal concentrating defect caused by central or renal lesions. In these patients, there is consistent polyuria associated with diluted urine, indicated by urine osmolality that can be lower than $200 \mathrm{mOsm} / \mathrm{kg} .{ }^{2}$ In diabetes insipidus, a water deprivation test will reveal increasing serum sodium without significant change in urine osmolality (Table 2). The central versus nephrogenic origin can be distinguished by good response to administered desmopressin in patients with a central defect. ${ }^{2}$ Central causes are similar to ones leading to hypodipsia and are a result of injury to the hypothalamus by surgery, traumatic brain injury, vascular occlusion, or hemorrhage. ${ }^{2}$ Nephrogenic causes are from drugs (lithium, foscarnet, amphotericin B, carbamazepine, or clozapine are some examples), hypercalcemia, hypokalemia, obstructive nephropathy, or renal failure (acute or chronic). ${ }^{2,23}$

Hypotonic fluid depletion can also result from insensible fluid losses or gastrointestinal losses. Significant insensible hypotonic fluid losses can result from fever, diaphoresis, or tachypnea. ${ }^{2}$ Common gastrointestinal fluid losses in the elderly include diarrhea, vomiting, nasogastric drainage, and use of osmotic agents such as lactulose. ${ }^{2}$ If hypotonic fluid loss is considered, as long as kidney function is intact, there should be a renal response to retain sodium; therefore, urine osmolality should be elevated and urine sodium level reduced. ${ }^{2}$ Age-related changes may limit the extent of the elevation in urine osmolality and the decrease in urine sodium.

Lastly, rarer causes of hypernatremia in the elderly population include sodium overload from iatrogenic excessive administration of sodium bicarbonate or hypertonic saline, primary hyperaldosteronism, or Cushing's syndrome. ${ }^{1}$

Any altered mental state, dementia or delirium, can cause a patient to decrease free water intake. Nursing home patients may be dependent on caretakers for adequate hydration. According to the Nursing Home Reform Act of 1987, these facilities can be held liable for inadequate nutrition and hydration of a patient because of neglect. ${ }^{28}$ In patients with hypernatremic dehydration resulting from neglect, there may be no laboratory abnormalities other than the hypernatremia, and the deficits may be corrected with appropriate measures. Institutions have the responsibility to monitor fluid intake.

\section{Conclusion}

Hypernatremia in the elderly is not unusual and is associated with significant morbidity and mortality. The geriatric population is predisposed to developing hypernatremia as a result of physiologic changes in thirst sensitivity, urinary concentrating ability, and total body water. The causes for hypernatremia may be elusive, and initial workup is essential to prevent delay in diagnosis and management. As in the case presented, it is essential to consider multiple causes of hypernatremia to prevent recurrence and significant morbidity and mortality and to prevent false accusations of substandard care.

\section{Disclosure}

The authors have no conflicts of interest in this work.

\section{References}

1. Adrogué HJ, Madias NE. Hypernatremia. N Engl J Med. 2000;342(20): 1493-1499.

2. AlZahrani A, Sinnert R, Gernsheimer J. Acute kidney injury, sodium disorders, and hypercalcemia in the aging kidney: diagnostic and therapeutic management strategies in emergency medicine. Clin Geriatr Med. 2013;29(1):275-319.

3. Himmelstein DU, Jones AA, Woolhandler S. Hypernatremic dehydration in nursing home patients: an indicator of neglect. $J$ Am Geriatr Soc. 1983;31(8):466-471.

4. Turgutalp K, Özhan O, Gök Oğuz E, et al. Community-acquired hypernatremia in elderly and very elderly patients admitted to the hospital: clinical characteristics and outcomes. Med Sci Monit. 2012; 18(12):CR729-CR734.

5. Arinzon Z, Feldman J, Peisakh A, Zuta A, Berner Y. Water and sodium disturbances predict prognosis of acute disease in long term cared frail elderly. Arch Gerontol Geriatr. 2005;40(3):317-326.

6. Darmon M, Timsit JF, Francais A, et al. Association between hypernatraemia acquired in the ICU and mortality: a cohort study. Nephrol Dial Transplant. 2010;25(8):2510-2515.

7. Hoorn EJ, Betjes MG, Weigel J, Zietse R. Hypernatraemia in critically ill patients: too little water and too much salt. Nephrol Dial Transplant. 2008;23(5):1562-1568.

8. Lindner G, Funk GC, Schwarz C, et al. Hypernatremia in the critically ill is an independent risk factor for mortality. Am J Kidney Dis. 2007;50(6):952-957.

9. Long CA, Marin P, Bayer AJ, Shetty HG, Pathy MS. Hypernatraemia in an adult in-patient population. Postgrad Med J. 1991; 67(789):643-645.

10. Molaschi M, Ponzetto M, Massaia M, Villa L, Scarafiotti C, Ferrario E. Hypernatremic dehydration in the elderly on admission to hospital. $J$ Nutr Health Aging. 1997;1(3):156-160.

11. Borra SI, Beredo R, Kleinfeld M. Hypernatremia in the aging: causes, manifestations, and outcome. J Natl Med Assoc. 1995;87(3):220-224.

12. Chassagne P, Druesne L, Capet C, Ménard JF, Bercoff E. Clinical presentation of hypernatremia in elderly patients: a case control study. J Am Geriatr Soc. 2006;54(8):1225-1230. 
13. Fitzsimons JT. Angiotensin, thirst, and sodium appetite. Physiol Rev. 1998;78(3):583-686.

14. Phillips PA, Rolls BJ, Ledingham JG, et al. Reduced thirst after water deprivation in healthy elderly men. $N$ Engl J Med. 1984;311(12): 753-759.

15. Phillips PA, Bretherton M, Johnston CI, Gray L. Reduced osmotic thirst in healthy elderly men. Am J Physiol. 1991;261(1 Pt 2):R166-R171.

16. Wright JW, Harding JW. The brain renin-angiotensin system: a diversity of functions and implications for CNS diseases. Pflugers Arch. 2013;465(1):133-151.

17. Lindeman RD, Van Buren HC, Raisz LG. Osmolar renal concentrating ability in healthy young men and hospitalized patients without renal disease. N Engl J Med. 1960;262(26):1306-1309.

18. Rowe JW, Shock NW, DeFronzo RA. The influence of age on the renal response to water deprivation in man. Nephron. 1976;17(4):270-278.

19. McAloon Dyke M, Davis KM, Clark BA, Fish LC, Elahi D, Minaker KL. Effects of hypertonicity on water intake in the elderly: an age-related failure. Geriatr Nephrol Urol. 1997;7(1):11-16.

20. O’Neill PA, McLean KA. Water homeostasis and ageing. Med Lab Sci. 1992;49(4):291-298.

21. Luft FC, Grim CE, Fineberg N, Weinberger MC. Effects of volume expansion and contraction in normotensive whites, blacks, and subjects of different ages. Circulation. 1979;59(4):643-650.
22. Beck LH. The aging kidney. Defending a delicate balance of fluid and electrolytes. Geriatrics. 2000;55(4):26-28.

23. Liamis G, Milionis H, Elisaf M. A review of drug-induced hyponatremia. Am J Kidney Dis. 2008;52(1):144-153.

24. Al-Absi A, Gosmanova EO, Wall BM. A clinical approach to the treatment of chronic hypernatremia. Am J Kidney Dis. 2012;60(6): 1032-1038.

25. Alshayeb HM, Showkat A, Babar F, Mangold T, Wall BM. Severe hypernatremia correction rate and mortality in hospitalized patients. Am J Med Sci. 2011;341(5):356-360.

26. Fourman P, Leeson PM. Thirst and polyuria, with a note on the effects of potassium deficiency and calcium excess. Lancet. 1959;1(7067):268-271.

27. Manitius A, Levitin H, Beck D, Epstein FH. On the mechanism of impairment of renal concentrating ability in potassium deficiency. J Clin Invest. 1960;39(4):684-692.

28. Breen GM, Matusitz J, Wan TT. The use of public policy analysis to enhance the Nursing Home Reform Act of 1987. Soc Work Health Care. 2009; 48(5):505-518.
Clinical Interventions in Aging

\section{Publish your work in this journal}

Clinical Interventions in Aging is an international, peer-reviewed journal focusing on evidence-based reports on the value or lack thereof of treatments intended to prevent or delay the onset of maladaptive correlates of aging in human beings. This journal is indexed on PubMed Central, MedLine,

\section{Dovepress}

CAS, Scopus and the Elsevier Bibliographic databases. The manuscript management system is completely online and includes a very quick and fair peer-review system, which is all easy to use. Visit http://www.dovepress. com/testimonials.php to read real quotes from published authors. 